\title{
Probiotic Properties of some Lactobacillus spp. that can survive in the Presence of Viral Gastroenteritis
}

\author{
Elmas Ceren Ikiz ${ }^{1}$, Gulcin Alp Avci ${ }^{(\mathbb{C}}$ Hande Alp $^{2}$ \\ ${ }^{1}$ Hitit University, Department of Molecular Biology and Genetics, Çorum, Turkey \\ ${ }^{2}$ Child Allery Specialist, Remzi Oguz Arik Neighborhood, Kavaklıdere Street, 23/2, Ankara, Turkey
}

\begin{abstract}
A B S T R AC T
/ iral acute gastroenteritis is a serious health problem worldwide with the high morbidity and mortality rates. In fact there is no available antiviral treatment for gastroenteritis alternatively, the use of probiotics on which have numerous benefits, has been on the increase recently. Therefore, to be able to identify the strains that can survive in viral acute gastroenteritis and their distinguishable features, seven strains of Lactobacillus spp. were isolated from the stool samples of $0-5$ year old children with viral gastroenteritis in this study. Strains were identified by API $50 \mathrm{CH}$ test. EPS production capacities, acid resistance, bile tolerance, antibiotic susceptibilities, and antimicrobial activities against Escherichia coli ATCC 25922 were determined in the order to investigate their probiotic features. Strains were characterized as Lactobacillus plantarum. All strains survived in De Man Rogosa and Sharpe (MRS) broth with adjusted $\mathrm{pH}$ values of 2 and 3, despite high inhibition rates (95.2-99.2\% and 98.3-99.2\%, respectively). Furthermore, all strains maintained their viability within MRS broth mediums that contain $0.15 \%, 0.2 \%$ and $0.3 \%$ bile (viability rates as $81.4-92.5 \%, 80.9$ $87.3 \%$ and $73.2-89.2 \%$, respectively). Exopolysaccharide production $(4.13-50.33 \mathrm{mg} / \mathrm{mL}$ ) was observed in all strains except for 182a. No antimicrobial activity was detected against E. coli ATCC 25922. All strains experienced high sensitivity to erythromycin while showing resistance to vancomycin. In conclusion, L. plantarum strains obtained in this study can be further investigated for describe other probiotic features and may be used for the production of new probiotic products to provide sufficient therapies in further studies.
\end{abstract}

Keywords:

Acute Gastroenteritis, Probiotics, Lactobacillus spp.

\section{INTRODUCTION}

G astroenteritis which can affect all age groups, especially children under the age of 5 , is an important health problem in both developed and developing countries worldwide with high mortality rate, causing socioeconomic burden $[1,2]$. It can be classified as acute, dysenteric or chronic. Acute gastroenteritis causing major outbreaks by viral, parasitic or bacterial agents has high morbidity and mortality all over the world as well as in Turkey. The most common etiologic factor of acute gastroenteritis is viral agents. Millions of diarrhea attacks and outpatient examinations are observing worldwide due to the viral gastroenteritis, and worse, more than 2 million children are hospitalized each year $[1,3]$. İn the lead of rotaviruses, viral gastroenteritis can also caused by different viral agents that include norovirus, adenovirus, bocavirus, sapovirus and astrovirus [4]. In viral gastroenteritis, fecal-orally transmitted virus particles cross over the gastrointestinal tract and bind to intestinal epithelium and cause inflammation in epithelial cells. As a result of inflammation-causing villous atrophy and mucosal damage, absorption of nutrients and fluids is impaired and the accumulated substances are excreted with severe diarrhea. In addition to diarrhea, acute gastroenteritis with fever, vomiting, nausea and abdominal pain lasts 1-2 weeks and causes significant loss of fluid [3, 4]. In order to overcome these diseases, especially in children's units, schools, elderly care centers, social areas and at home, hygienic cautions, carefully washing of the nutrients, well-balanced diet and vaccines are recommended. There is no available vaccine produced against rotavirus and other viral agents, except Rotarix and Rotateq in which United States has been using as therapeutics [2]. Viral gastroenteritis is a self-limiting disease with no effective antiviral drug treatment in use. The main treatment is to provide recovery of lost fluid and electrolytes. To restore intestinal flora 
balance, recent studies have focused on dietary supplementation of probiotics that have many health-beneficial effects, such as cancer prevention, immune system regulation, assistance to lactose digestion, regulation of intestinal flora, heart disease prevention, and lowering serum cholesterol levels $[1,5,6]$. Because of breast milk is the main source of probiotic and prebiotic nutrition, it is recommended that, breastfeeding should be more often for the homeostasis of intestine and meals per day of babies fed by supplementary food rather than mother's milk should be increased. Several probiotics such as Bifidobacterium bifidum, Streptococcus thermophilus, Lactobacillus reuteri, Lactobacillus casei and Lactobacillus rhamnosus (GG origin) have been reported to be useful in gastroenteritis diarrhea $[7,8]$, however, those are still limited. We hypothesize that probiotic bacteria are able to survive and maintain colonization, possibly by developing new features or improving already existed ones under all stress conditions mentioned above caused by viral acute gastroenteritis. This is a new insight of our study revealed because in literature most of researchers have based their study on the strains isolated from healthy individuals and fermented products. Therefore, the first objective of this study was to isolate lactobacilli that could survive in the presence of viral acute gastroenteritis agents. The second aim of this study was to determine some probiotic properties of these lactobacilli.. Strains obtained from this study may be use in future research with purpose of developing new probiotic products or using as supplements in nutritional therapies.

\section{MATERIAL AND METHODS}

\section{Isolation and Identication of Bacteria}

In our study, 51 stool samples previously determined as rotavirus positive (18), adenovirus positive (17), adenovirus/rotavirus positive (2) and negative (14) by ELISA method were used. Lactobacilli strains were isolated by culture method from 10\% Phosphate-Buffered Saline (PBS, pH:7,4) diluted stool samples. For isolation, incubation was provided in MRS Broth (Merck, Darmstadt, Germany) and MRS-Agar mediums (pH $6.8 \pm 0.02)$ at $37^{\circ} \mathrm{C}$ for $18-24$ hours in an anaerobic environment. MRSAgar mediums were prepared by adding 1,5\% Agar-agar (Merck, Darmstadt, Germany). Anaerobic environment was obtained by Anaerocult ${ }^{\circledR} \mathrm{C}$ (Merck, Darmstadt, Germany) kit. The strains were stored in $60 \%$ glycerol at $80^{\circ} \mathrm{C}$.

Classical and biochemical identification methods were used for Lactobacillus strains. Colony morphology of bacteria was observed and their microscopic morphology and response to gram staining were examined. $\mathrm{API}^{\oplus} 50 \mathrm{CH} /$ CHL (API System, Bio-Merieux, France) tests were applied for the identification of biochemical bacteria.

\section{Determination of Some Probiotic Properties}

\section{Exopolysaccharide Production of Isolates}

The method obtained from literature is used for EPS production [9]. In spectrophotometer with $600 \mathrm{~nm}$ wavelength, the Optical Density 600nm values of active Lactobacillus strains were adjusted to 0.600. Strains $\left(\mathrm{OD}_{600} \sim 0,600\right)$ were inoculated in new MRS medium at $2 \%$ rate and incubated at $37^{\circ} \mathrm{C}$ in the anaerobic environment for 24 hours. After 24 hours, each $1 \mathrm{~mL}$ of active cultures was boiled in sterile eppendorf tubes at $96{ }^{\circ} \mathrm{C}$ for 10-15 minutes and allowed to cool. Samples were centrifuged at $13000 \mathrm{rpm}$ for $25 \mathrm{~min}$ with an addition of $170 \mu \mathrm{l}$ 85\% Trichloroacetic acid (Merck, Darmstadt, Germany). The supernatants were again centrifuged by adding an equal volume of ethanol in the new sterile eppendorf tubes. For the second time, ethanol was added onto the pellets, then, dissolved and centrifuged again to precipitate.

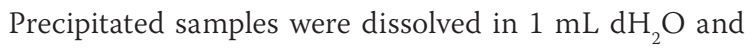
the amount of EPS was determined using phenol-sulfuric acid method [10]. To determine the amount of EPS production, the standard curve was extracted using glucose solutions ranging from 5 to $100 \mathrm{mg} / \mathrm{L}$.

\section{Tolerance to Acid and Bile of Isolates}

In order to determine the reproduction rate of lactobacilli in acidic medium, MRS media were prepared as $\mathrm{pH}$ 2.0, pH 3.0, pH 6.8 (control) and pH 8.0. Mediums' pHs have adjusted by $\mathrm{pH}$ meter (with $1 \mathrm{~N} \mathrm{NaOH}$ and $1 \mathrm{~N}$ $\mathrm{HCl}$ ). All strains were activated twice before experiment to obtain cultures in logarithmic phase. Twice activated Lactobacillus spp. strains were incubated in the MRS medium with different $\mathrm{pHs}$ at $37^{\circ} \mathrm{C}$ in anaerobic media for 24 hours. After 24 hours, the density of the cultures was measured by spectrophotometer set to a wavelength of $600 \mathrm{~nm}$.

To characterize the resistance of isolated strains to bile; MRS media containing 0\% (control), 0.15\%, 0.2\% and 0.3\% bile salt was prepared and twice activated Lactobacillus spp. were inoculated in these media $\left(\mathrm{OD}_{600}=0.600\right)$, after incubation at $37^{\circ} \mathrm{C}$ in anaerobic media for 24 hours, the intensity of cultures was measured by spectrophotometer $(600 \mathrm{~nm})$.

\section{Resistance to Antibiotics of Isolates}

Disc diffusion method was preferred to analyze whether strain resists to antibiotics. Twice activated Lactobacillus spp. cultures' OD values at $600 \mathrm{~nm}$ were adjusted to 0.600 . Each $100 \mu \mathrm{l}$ amount of cultures was inoculated upon Muller-Hinton Agar medium, and then, antibiotic discs (penicillin G, vancomycin, chloramphenicol, azithromycin, tetracycline, ampicillin, gentamicin, clindamycin, erythromycin, amikacin) were placed. The diameters of the inhibition zones formed after 24 hours of the anaerobic incubation at $37^{\circ} \mathrm{C}$ were measured. 


\section{Antimicrobial Activity against E. coli ATCC 25922 of Isolates}

Disc diffusion method was used to determine the antimicrobial activity of the strains. Twice activated Lactobacillus spp. strains and E. coli ATCC 25922 cultures' OD values at $600 \mathrm{~nm}$ were set to $0.600 .100 \mu \mathrm{l}$ of E. coli culture were inoculated upon Muller-Hinton Agar media (Merck, Darmstadt, Germany), and sterile discs that prepared from Watmann No: 4 filter papers were placed. $5 \mu \mathrm{l}$, $10 \mu \mathrm{l}$ and $15 \mu \mathrm{l}$ of Lactobacillus spp. strains were added onto the prepared sterilized discs, and petri dishes were incubated at $37^{\circ} \mathrm{C}$ for 24 hours in anaerobic media. After 24 hours, the inhibition zone diameters were measured.

\section{Statistical Analysis}

In all studies, the mean values of two different parallels are given. SPSS 20.0 (SPSS Inc., Chicago) program was used for statistical analysis. According to the one-way ANOVA correlation, the relationships between EPS production and resistance to different pHs as well as EPS production and bile tolerance were investigated.

\section{RESULTS}

\section{Isolation and Identification of Bacteria}

The seven Lactobacillus spp. were isolated from 51 stool samples. One (66a) of these strains was isolated from viral negative patient, and the rest (87a, 87b, 182a, 182b, 183a and 209a) was isolated from rotavirus positive samples. Lactobacillus spp. strains could not be isolated from adenovirus positive samples. Microscopic and colony morphologies of isolated strains are shown in the Fig. 1.

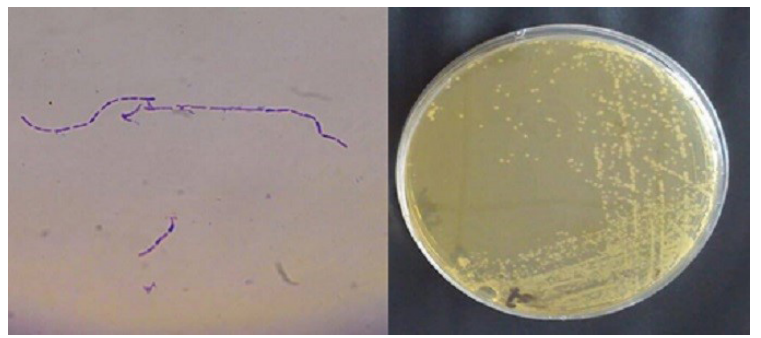

Figure 1. Microscopic image and colony morphology of Lactobacillus strains

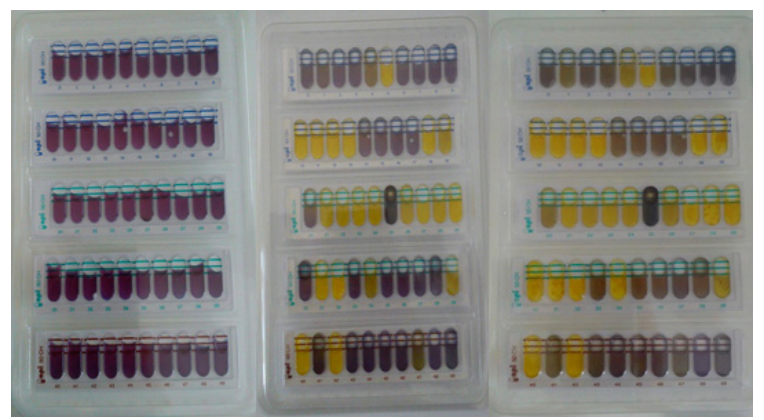

Figure 2. API test results (from left to the right ; after $0 \mathrm{~h}, 24 \mathrm{~h}$ and 48 h) ; Yellow color was described as positive result, purple color as negative result and intermediate color as pseudo positive result.
Table 1. API $50 \mathrm{CH}$ identification test results of isolates.

\begin{tabular}{cc}
\hline $\begin{array}{c}\text { Strains } \\
\text { codes }\end{array}$ & $\begin{array}{c}\text { API results (\% Identification } \\
\text { rates) }\end{array}$ \\
$66 \mathrm{a}$ & L.plantarum $(99,9 \%)$ \\
$87 \mathrm{a}$ & L.plantarum $(99,8 \%)$ \\
$87 \mathrm{~b}$ & L.plantarum $(98,9 \%)$ \\
$182 \mathrm{a}$ & L.plantarum $(99,9 \%)$ \\
$182 \mathrm{~b}$ & L.plantarum $(99,9 \%)$ \\
$183 \mathrm{a}$ & L.plantarum $(99,9 \%)$ \\
$209 \mathrm{a}$ & L.plantarum $(99,3 \%)$
\end{tabular}

API $50 \mathrm{CH}$ identification test results of the 7 Lactobacillus strains are given in Fig. 2 and Table 1.

\section{Determination of Some Probiotic Properties Exopolysaccharide Production of Isolates}

The EPS production of the samples is given in Table 2. The strain showing the highest EPS production in

L. plantarum 182b strain, but EPS production was not detected in the 182a strain. EPS production capacity determination studies for isolated and identified strains were performed in two different time intervals. Due to the variable results in measurements, statistically standard deviation could not be calculated.

Table 2. The Exopolysaccharide production amount of isolated strains

$\begin{array}{cc}\text { Strain Codes } & \text { EPS Production }(\mathrm{mg} / \mathrm{L}) \\ \text { L. plantarum } 66 \mathrm{a} & 5.54 \\ \text { L. plantarum } 87 \mathrm{a} & 9.17 \\ \text { L. plantarum } 87 \mathrm{~b} & 7.93 \\ \text { L.plantarum 182a } & \mathrm{ND} \\ \text { L.plantarum 182b } & 50.33 \\ \text { L.plantarum 183a } & 4.13 \\ \text { L.plantarum 209a } & 9.01 \\ \text { L. plantarum DSM 20174 } & 132.0\end{array}$

${ }^{a}$ : L. plantarum DSM 20174; Reference strain

\section{Tolerance to Acid and Bile of Isolates}

The viability measurements of seven strains in MRS media adjusted $\mathrm{pH}$ 2.0, $\mathrm{pH}$ 3.0, pH 6.8 (control) and pH 8.0 are shown in Table 3. It was indicated that seven

L. plantarum strains were also active at $\mathrm{pH} 2.0$ according to the viability measurements in different acidic environments.

In medium without bile and with $0.15 \%, 0.2 \%$ and $0.3 \%$ bile, viability measurements for seven strains are given in Table 4. As a result of bile tolerance measurement, seven strains experienced less growth in the media with bile than control. The strains that possessed the highest and lowest bile tolerance were characterized as $66 \mathrm{a}$ and 209a, respectively. 
Table 3. Acid resistance of isolated strains in different $\mathrm{pHs}$

\begin{tabular}{ccccc}
\hline \multirow{2}{*}{ Strain Codes } & \multicolumn{4}{c}{ Acid resistance } \\
\cline { 2 - 5 } & $\mathrm{pH} 2.0$ & $\mathrm{pH} 3.0$ & $\mathrm{pH} 6.8^{\mathrm{a}}$ & $\mathrm{pH} 8.0$ \\
L. plantarum 66a & $0.11 \pm 0.08$ & $0.02 \pm 0.01$ & $2.33 \pm 0.00$ & $2.25 \pm 0.01$ \\
L. plantarum 87a & $0.07 \pm 0.11$ & $0.04 \pm 0.00$ & $2.33 \pm 0.08$ & $2.27 \pm 0.20$ \\
L. plantarum 87b & $0.03 \pm 0.01$ & $0.03 \pm 0.01$ & $2.33 \pm 0.36$ & $2.81 \pm 0.03$ \\
L.plantarum 182a & $0.02 \pm 0.01$ & $0.02 \pm 0.01$ & $2.44 \pm 0.17$ & $2.41 \pm 0.06$ \\
L. plantarum 182b & $0.04 \pm 0.01$ & $0.02 \pm 0.01$ & $2.50 \pm 0.03$ & $1.25 \pm 0.04$ \\
L.plantarum 183a & $0.04 \pm 0.01$ & $0.04 \pm 0.01$ & $2.46 \pm 0.02$ & $2.53 \pm 0.02$ \\
L. plantarum 209a & $0.02 \pm 0.00$ & $0.03 \pm 0.02$ & $2.22 \pm 0.02$ & $2.26 \pm 0.10$ \\
L. plantarum & $0.24 \pm 0.20$ & $0.38 \pm 0.10$ & $3.52 \pm 0.50$ & $3.46 \pm 0.34$ \\
$\quad$ DSM 20174 & & & \\
pH 6.8 & & & \\
L. : Control medium & & &
\end{tabular}

Table 4. Viability measurements of isolated strains in different bile concentration

\begin{tabular}{ccccc}
\hline \multirow{2}{*}{ Strain Codes } & \multicolumn{4}{c}{ Bile tolerance } \\
\cline { 2 - 5 } & $0 \%^{\mathrm{a}}$ & $0.15 \%$ & $0.20 \%$ & $0.30 \%$ \\
L. plantarum 66a & $2.33 \pm 0.00$ & $2.04 \pm 0.04$ & $2.04 \pm 0.02$ & $2.09 \pm 0.08$ \\
L. plantarum 87a & $2.33 \pm 0.08$ & $1.97 \pm 0.05$ & $1.96 \pm 0.01$ & $1.96 \pm 0.10$ \\
L. plantarum 87b & $2.33 \pm 0.36$ & $1.89 \pm 0.01$ & $1.93 \pm 0.01$ & $1.99 \pm 0.07$ \\
L.plantarum 182a & $2.44 \pm 0.17$ & $2.02 \pm 0.00$ & $2.01 \pm 0.01$ & $1.86 \pm 0.26$ \\
L.plantarum 182b & $2.50 \pm 0.03$ & $2.07 \pm 0.17$ & $2.02 \pm 0.05$ & $2.15 \pm 0.10$ \\
L. plantarum 183a & $2.46 \pm 0.02$ & $2.04 \pm 0.03$ & $2.01 \pm 0.08$ & $1.85 \pm 0.06$ \\
L. plantarum 209a & $2.22 \pm 0.02$ & $2.06 \pm 0.05$ & $1.86 \pm 0.01$ & $1.63 \pm 0.03$ \\
L. plantarum DSM & $3.52 \pm 0.50$ & $3.82 \pm 0.10$ & $3.18 \pm 0.05$ & $1.02 \pm 0.02$ \\
20174 & & & & \\
a. bile free & & & & \\
b: Reference Strain & & & &
\end{tabular}

\section{Resistance to Antibiotics of Isolates}

Seven isolated strains of $L$. plantarum were resistant to vancomycin. While the strain 87 a was also showing resistance to tetracycline; the strain $87 \mathrm{~b}$ experienced resistance to penicillin and tetracycline. The strains 182a and 209a showed resistance to penicillin beside vancomycin. Overall, all strains presented susceptibility to clindamycin, azithromycin, ampicillin, gentamicin, erythromycin and chloramphenicol. The highest sensitivity of all strains was measured for erythromycin. The most resistant strains against 10 antibiotics measured were strains 87 a and $87 \mathrm{~b}$. The least resistant strains were the 182a and $182 \mathrm{~b}$ strains. Inhibition zone measurements are given in Table 5.

\section{Antimicrobial Activity against E. coli ATCC 25922 of isolates}

The antimicrobial effect on E.coli ATCC 25922 was not observed in three different concentrations $(5 \mu \mathrm{l}, 10 \mu \mathrm{l}$ and $15 \mu \mathrm{l}$ ) of seven L. plantarum strains.

\section{DISCUSSION}

The formation of intestinal microbiota begins with birth.
The population of microorganisms in the baby's intestine reaches up to $10^{9}-10^{10}$ of bacteria rising from the environment of contact with such as doctors, mother and other individuals, mother's milk and nutrients in the first days of post-natal $[11,12]$. The imbalances in dietary habits, drug use and diseases lead to the change of natural intestinal microbiota in humans, and thus, to an imbalance in the nutrient absorption and dehydration. An important factor in the change of intestinal flora is acute gastroenteritis (AGE) infections in the early stages of life [13-15].

As one from the negative patient and six from the rotavirus positive patients, seven gram (+) bacilli strains were isolated. No isolation from adenovirus positive samples could be obtained. It is thought that cell lysis caused by adenoviruses is an important obstacle in adhesion of bacteria. Thus, the survival rates of not adhesive bacteria are decreased significantly.

The classical identification methods and API $50 \mathrm{CHL}$ test results revealed that 7 of these strains belonged to the Lactobacillus plantarum strains. Most of the studies have been based on the isolation of probiotic bacteria from healthy individuals [16-18]. In our study, unlike literature, probiotic strains that can sustain their viability despite the changing conditions and inflammation were isolated from patients with AGE. Therefore in the literature, species diversity is higher in isolation and isolation success has been higher. This arises from the increasingly difficulties in bacterial colonization due to epithelial damage, as well as the excretion of bacteria with excrement due to diarrhea [16-18].

In order to overtake the gastrointestinal tract and colonize the intestine when taken orally; the probiotics need to survive in the acidic environment of the stomach, in the high bile concentration of the intestine, and in the presence of a wide variety of pathogens $[19,20]$. For this purpose, in vitro imitations of these conditions were prepared and acid resistance and bile tolerance of isolated strains were investigated.

All isolates were determined to have high inhibition at $\mathrm{pH}$ 2.0, but they were able to maintain their viability. Strains' rate of decrease at pH 2.0 compared with pH 6.8 (control) varied between $95.2 \%$ and $99.2 \%$. The decrease rate of strains at $\mathrm{pH} 3.2$ according to the control ranged from $98.3 \%$ to 99.2\%. The strain with the highest tolerance to the acidic medium was determined as L. plantarum 66a while the lowest tolerance was characterized as L. plantarum 182a. In the literature, lactobacilli strains with high inhibition in acidic environment have been reported [18, 21-24], and particularly in one study researchers concluded that the strains could not maintain their viability in the acidic environment [18]. In our study, despite the presence of AGE, all strains 
Table 5. Antibiogram test results of strains ( $\mathrm{mm}$ zone diameter)

\begin{tabular}{|c|c|c|c|c|c|c|c|c|c|c|}
\hline \multirow{2}{*}{ Strain Codes } & \multicolumn{4}{|c|}{ Bile tolerance } & \multirow[b]{2}{*}{$\mathrm{AK}^{\mathrm{e}}$} & \multirow[b]{2}{*}{$C^{f}$} & \multirow[b]{2}{*}{$\mathrm{E}^{\mathrm{g}}$} & \multirow[b]{2}{*}{$\mathrm{TE}^{\mathrm{h}}$} & \multirow[b]{2}{*}{$\mathrm{CN}^{\mathrm{i}}$} & \multirow[b]{2}{*}{$\mathrm{VA}^{\mathrm{k}}$} \\
\hline & $\mathrm{DA}^{\mathrm{a}}$ & $\mathrm{AZM}^{\mathrm{b}}$ & $\mathrm{P}^{\mathrm{c}}$ & $\mathrm{AMP}^{\mathrm{d}}$ & & & & & & \\
\hline L. plantarum 66a & $25.0 \pm 0.0$ & $28.4 \pm 1.2$ & $15.6 \pm 0.2$ & $22.4 \pm 0.5$ & $15.0 \pm 0.0$ & $24.0 \pm 0.3$ & $22.3 \pm 1.1$ & $12.6 \pm 1.6$ & $17.4 \pm 1.6$ & $\mathrm{NIZ}^{\mathrm{m}}$ \\
\hline L. plantarum 87a & $17.9 \pm 0.2$ & $20.6 \pm 0.5$ & $13.2 \pm 0.2$ & $20.8 \pm 1.1$ & $17.3 \pm 1.1$ & $18.8 \pm 0.7$ & $22.0 \pm 0.0$ & NIZ & $14.9 \pm 0.2$ & NIZ \\
\hline L. plantarum 87b & $17.4 \pm 0.5$ & $20.1 \pm 2.6$ & NIZ & $23.4 \pm 0.6$ & $15.0 \pm 1.4$ & $21.3 \pm 1.7$ & $29.5 \pm 1.1$ & NIZ & $20.3 \pm 0.3$ & NIZ \\
\hline L. plantarum 182a & $16.0 \pm 0.0$ & $27.6 \pm 4.4$ & NIZ & $21.5 \pm 1.2$ & $22.6 \pm 1.6$ & $24.1 \pm 0.2$ & $27.0 \pm 0.0$ & $12.9 \pm 1.9$ & $23.9 \pm 1.6$ & NIZ \\
\hline L. plantarum $182 \mathrm{~b}$ & $20.2 \pm 1.1$ & $26.3 \pm 1.8$ & $13.4 \pm 0.7$ & $14.8 \pm 0.7$ & $24.1 \pm 1.6$ & $28.0 \pm 1.1$ & $31.1 \pm 0.6$ & $14.8 \pm 3.9$ & $21.4 \pm 0.5$ & NIZ \\
\hline L. plantarum 183a & $20.0 \pm 0.7$ & $25.5 \pm 0.3$ & $12.7 \pm 1.8$ & $20.4 \pm 0.5$ & $17.8 \pm 1.1$ & $15.5 \pm 0.0$ & $24.1 \pm 0.2$ & $12.7 \pm 0.1$ & $17.1 \pm 0.9$ & NIZ \\
\hline L. plantarum 209a & $14.1 \pm 0.8$ & $15.9 \pm 1.2$ & NIZ & $22.4 \pm 0.9$ & $18.6 \pm 0.5$ & $21.4 \pm 0.7$ & $26.5 \pm 1.4$ & $10.0 \pm 0.0$ & $20.6 \pm 1.1$ & NIZ \\
\hline
\end{tabular}

were able to maintain their presence in low acidity.

The viability of strains decreased as the concentration of bile increased. The decrease rate of cultures in $0.3 \%$ bile concentration was compared with the control with no bile varied between $10.2 \%$ and $26.8 \%$. The strain with the hig hest tolerance was determined as L. plantarum 66a when the strain with the lowest tolerance was determined as L. plantarum 209a. In some studies investigating the resistance of lactobacilli strains isolated from healthy babies to high bile salt concentrations, a high rate of inhibition was reported in the $0.3 \%$ concentration of bile salt [18, 21-26]. The viability of $L$. plantarum strains examined in our study has decreased, parallel to the most studies in the literature.

Exopolysaccharides are structures those are produced by the bacterium, which are found in the cell wall and bind the bacteria to the cell. These saccharides protect the cells against antibiotics and toxic substances, support immunity, and thus have antitumoral and cholesterol-lowering activity $[27,28]$. The probiotic bacteria have an importance, for the colonization race with pathogen ones and, are capable of producing EPS which is physically protective and enhance the adhesion to the intestine. Therefore, it is highly preferred for a probiotic bacterium that the high EPS production capacity. In this study, EPS production capacities of isolated 7 strains were investigated and EPS production was measured in all strains except L. plantarum 182a. The EPS values measured in the rest 6 L. plantarum strains ranged from 4.13 to $50.33 \mathrm{mg} / \mathrm{mL}$. Some of researchers reported that the range of EPS production of probiotic strains isolated from fermented products and human feces was 102-263 mg/L [29]; while others noted this range for kefir bacteria as 173-378 mg/L [21]. Furthermore, in another study the EPS production range of lactic acid bacteria isolated from children feces age of 4-15 years have reported as 70-290 mg/L [18] and different researchers having investigated the probiotic properties of Lactobacillus strains found the range of EPS production as 62-287 mg/L [30]. In our study with compared to the literature, six of the L. plantarum strains showed low EPS production, while one showed no production. We claimed that the differences between the each measurement might be due to the source of the strains, the incubation conditions and the medium content. In our study, although the strains were isolated from an important infection condition called viral acute gastroenteritis, they were able to maintain their EPS production capability and the vitality in the intestine, even if there is a smaller amount of EPS production.

Since the study of replica within each group may potentially affect the significance of statistical analysis. There was no significant relationship between the EPS production of the strains and their resistance in acidic environments ( $p>0.05$ ). There was no significant correlation between EPS production of strains and tolerance to different bile concentrations too $(p>0.05)$. This was thought to be due to fluctuations in EPS production capacity. Statistically, there was a positive correlation between the acid resistance and the bile tolerance of the strains when resistance and tolerance of the strains were evaluated together $(\mathrm{p}<0.05)$.

Although probiotic bacteria remained viable throughout the gastrointestinal tract and colonized in the intestine, they need to be strong against further threats including the use of antibiotics to sustain this colonization and conserve their presence in the flora $[19,20]$. In addition, the transmission of antibiotic resistance genes to pathogenic bacteria from food bacteria and the proliferation of resistant pathogens in intestine are concerned as an important health issue. The inhibition effects of probiotics that possess antibiotic resistance genes, as well as their metabolites on pathogenic bacteria have recently been used strategically to prevent or treat infections such as acute gastroenteritis $[19,20]$. A probiotic bacterium should at least be resistant to common antibiotics and must have antimicrobial activity on pathogenic bacteria [19, 20]. In our study, all Lactobacillus plantarum strains showed resistance to vancomycin. Overall, all strains showed susceptibility to clindamycin, azithromycin, ampicillin, gentamicin, erythromycin, and chloramphenicol. The antibiotic with the highest sensitivity of all strains was characterized as erythromycin. European Committee on Antimicrobial Susceptibility Testing (EU- 
CAST) antibiotic resistance assessment data did not define the inhibition zone range in the disk diffusion method for gram-positive anaerobe bacteria. The resistant/sensitive terms mentioned in our study were used according to the zone formation and the size of the inhibition zone. Measurements of resistance/susceptibility of strains by EUCAST Minimum inhibitory concentration (MIC) method can be supported by further studies. In the literature, resistance of lactobacilli strains isolated from healthy babies to various antibiotics has been investigated. In our study, similar to literature, all

L. plantarum strains experienced high sensitivity to erythromycin while showing resistance to vancomycin. Different results have been observed in different strains against other 8 used antibiotics $[18,26$, 32-34].

One of the important features of probiotic bacteria is that they can inhibit pathogenic bacteria by producing $\mathrm{HO}$, lactic acid, and various bacteriocins, and also, they can maintain flora balance $[19,20]$. In our study, antimicrobial activity of isolated lactobacilli strains on pathogen E. coli ATCC 25922 was investigated by disk diffusion method and no formation of the inhibition zone was observed. The inhibition effect of lactobacilli strains against various pathogens has been criticized in many studies and different results have been reported. In a study, different Lactobacillus species such as L. pentosus, L. casei spp rhamnosus, L. paracasei, L. casei, L. plantarum and L. fermentum showed inhibition effects against $E$. coli ATCC with zone size 4 to10 mm [18]. Another study reported inhibition zone sizes of

L. plantarum strains against E. coli as $16-20 \mathrm{~mm}$ [19]. While some researchers reported inhibition zone sizes of isolated strains against $E$. coli as 11,3-14,8 mm, others noted as 0-23 mm [22, 35]. In our studies, the antimicrobial effect on E.coli ATCC 25922 was not observed in three different concentrations $(5 \mu \mathrm{l}, 10 \mu \mathrm{l}$ and $15 \mu \mathrm{l})$ of seven L. plantarum strains.

\section{CONCLUSION}

The first objective of this study was to isolate lactobacilli that could survive in the presence of viral acute gastroenteritis agents. The second aim of this study was to determine some probiotic properties of these lactobacilli.As a result, it was observed that some L. plantarum strains were able to maintain their colonization in the intestine, despite of the viral infection and diarrhea. The some strains were able to maintain their viability in acidic environment, and even in media with bile salt. For this reason, when taken orally, the probiotic strains will be able to colonize in the intestine maintaining their viability in certain proportions, within acidic conditions of stomach as well as in bile condition of gut. Also, it was observed that the strains were resistant to vancomycin. In the works that we plan to do later, we aim to investigate some probiotic properties such as to adhere to intestinal cells, production of some secondary metabolites, cholesterol removal and bile salt deconjugation.

\section{ACKNOWLEDGEMENT}

This study was supported by University of Hitit, Department of Scientific Research Projects (Project No. FEF19004.17.004)

\section{REFERENCES}

1. Anderson EJ. Prevention and treatment of viral diarrhea in pediatrics. Expert Reviews 8 (2010) 205-217.

2. Donà D, Mozzo E, Scamarcia A, Picelli G, Villa M, Cantarutti L, Giaquinto C. Community-Acquired Rotavirus Gastroenteritis Compared with Adenovirus and Norovirus Gastroenteritis in Italian Children: A Pedianet Study. International Journal of Pediatrics (2016) 5236243.

3. Wilhelmi I, Roman E, Sanchez-Fauquier A. Viruses causing gastroenteritis. Clinical Microbiology and Infection 9 (2003) 247262.

4. Dashti AS, Ghahremani P, Hashempoor T, Karimi A. Molecular Epidemiology of Enteric Adenovirus Gastroenteritis in under-FiveYear-Old Children in Iran. Gastroenterology Research and Practice (2016) 2045697.

5. De Angelis M, Calasso M, Cavallo N, Di Cagno R, Gobbetti M. Functional proteomics within the genus Lactobacillus. Proteomics 16 (2016) 946-62.

6. Gonzalez-Ochoa G, Flores-Mendoza LK, Icedo-Garcia R, GomezFlores R, Tamez-Guerra P. Modulation of rotavirus severe gastroenteritis by the combination of probiotics and prebiotics. Archives in Microbiology 199 (2017) 953-961.

7. Grandy G, Medina M, Soria R, Araya M. Probiotics in the treatment of acute rotavirus diarrhea. A randomized, double- blind, controlled trial using two different probiotics preparations in Bolivian children. Biomed Center: Infectious Diseases (2010) 253.

8. Vandenplas Y. 2016. Probiotics and prebiotics in infectious gastroenteritis. Best Practice \& Research Clinical Gastroenterology 30 (2016) 49-53.

9. Valerie MM, Rawson HL. Effects of exopolysaccharide-producing strains of thermophilic lactic acid bacteria on the texture of stirred yoghurt. International Journal of Food Science and Technology 34 (1999) 137-143.

10. Dubois M, Gilles KA, Hamilton JK, Peters PA, Smith F. Colorimetric method for determination of sugars and related substances. Analytical Chemistry 28 (1956) 350-356.

11. Luckey TD, Floch MA. Introduction to intestinal microbiology. American Journal of Clinical Nutrition 25 (1972) 1291-1295.

12. Collins MD, Ribson GR. Probiotics, prebiotics and Synbiotics: Approaches for modulating the microbial ecology of the gut. American Journal of Clinical Nutrition 69 (1999) 1052-1057.

13. Arrieta MC, Stiemsma LT, Amenyogbe N, Brown EM, Finlay B. The intestinal microbiome in early life: health and disease. Frontiers in Immunology 5 (2014) 427.

14. Arboleya S, Watkins C, Stanton C, Ross RP. Gut Bifidobacteria populations in human health and aging. Frontiers in Microbiology 7 (2016) 1204.

15. Chen ST, Tsai CN, Lee YS, Lin CY, Huang KY, Chao HC, Lai MW, Chiu $\mathrm{CH}$. Intestinal microbiome in children with severe and complicated acute viral gastroenteritis. Scientific reports 7 (2017) 
46130 .

16. Jacobsen CN, Nielsen VR, Hayford AE, Moller PL, Michaelsen KF, Pærregaard A, Sandström B, Tvede M, Jakobsen M. Screening of Probiotic Activities of Forty-Seven Strains of Lactobacillus spp by in vitro Techniques and Evaluation of the Colonization Ability of Five Selected Strains in Humans. Applied and Environmental Microbiology 65 (1999), 4949-4956.

17. Martin R, Jimenez E, Olivares M, Marin ML, Fernandez L, Xaus J, Rodriguez JM. Lactobacillus salivarius CECT 5713, a potential probiotic strain isolated from infant feces and breast milk of a mother-child pair. International Journal of Food Microbiology 112 (2006) 35-43.

18. Tulumoglu Ş, Yuksekdag ZN, Beyatli Y, Şimsek Ö, Çınar B, Yaşar E. Probiotic properties of lactobacilli species isolated from children's feces. Anaerobe 24 (2013) 36-42.

19. Kaushik JK, Kumar A, Duary RK, Mohanty AK, Grover S, Batish VK. Functional and probiotic attributes of an indigenous isolate of Lactobacillus plantarum. Plosone 4 (2009) e8099.

20. Annuk H, Shchepetova J, Kullisaar T, Songisepp E, Zilmer M Mikelsaar M. Characterization of intestinal lactobacilli as putative probiotic candidates. Journal of Applied Microbiology 94 (2003) 403-412.

21. Sabir F, Beyatli Y, Cokmus C, Onal-Darilmaz D. Assessment of potential probiotic properties of Lactobacillus spp., Lactococcus spp., and Pedicoccus spp. Strains isolated from Kefir. Journal of Food Science 75 (2010)

22. Shokryazdan P, Sieo CC, Kalavathy R, Liang JB, Alitheen NB, Faseleh Jahromi M, Ho YW. Probiotic Potential of Lactobacillus Strains with Antimicrobial Activity against Some Human Pathogenic Strains. BioMed Research International (2014) 927268.

23. Verdenelli MC, Ghelfi F, Silvi S, Orpianesi C, Cecchini C, Cresci A. Probiotic properties of Lactobacillus rhamnosus and Lactobacillus paracasei isolated from human faeces. European Journal of Nutrition 48 (2009) 355-363.

24. Nawaz M, Wang J, Zhou A, Ma C, Wu X, Xu J. Screening and characterization o new potentially probiotic lactobacilli from breast-fed healthy babies in Pakistan. African Journal of Microbiology Research 5 (2011) 1428-1436.

25. Papamanoli E, Tzanetakis N, Litopoulou-Tzanetaki E, Kotzekidou P. Characterization of lactic acid bacteria isolated from a Greek dryfermented sausage in respect of their technological and probiotic properties. Meat Science (2003) 859-867.
26. Kotsou MG, Mitsou EK, Oikonomou IG, Kyriacou AA. In vitro assessment of probiotic properties of Lactobacillus strains from infant gut microflora. Food Biotechnology 22 (2008) 1-17.

27. Nikolic M, Lopez P, Strahinic I, Suarez A, Kojic M, Fernandez-Garcia M, Topisirovic L, Golic N, Ruas- Madiedo P. Characterization of the exopolysaccharide (EPS)- producing Lactobacillus paraplantarum BGCG11 and its non-EPS producing derivative strains as potential probiotics. International Journal of Food Microbiology 158 (2012) 155-162.

28. Caggianiello G, Kleerebezem M, Spano G. Exopolysaccharides produced by lactic acid bacteria: from health-promoting benefits to stress tolerance mechanisms. Applied Microbiology Biotechnology 100 (2016) 3877-86.

29. Aslim B, Yüksekdağ ZN, Beyatli Y, Mercan N. Exopolysaccharide production by Lactobacillus delbruckii subsp. bulgaricus and Streptococcus thermophilus strains under different growth conditions. World Journal of Microbiology \& Biotechnology 21 (2005) 673-677.

30. Ren D, Li C, Qin Y, Yin R, Du S, Ye F, Liu C, Liu H, Wang M, L Y, Sun Y, Li X, Tian M, Jin N. In vitro evaluation of the probiotic and functional potential of Lactobacillus strains isolated from fermented food and human intestine. Anaerobe 30 (2014) 1-10.

31. EUCAST Disk Diffusion Method for Antimicrobial Susceptibility Testing - Version 7.0 (January 2019) http://www.eucast. org/fileadmin/src/media/PDFs/EUCAST_files/Disk_test_ documents/2019_manuals/Manual_v_7.0_EUCAST_Disk_ Test_2019.pdf

32. Temmerman R, Pot B, Huys G, Swings J. Identification and Antibiotic Susceptibility of Bacterial Isolates from Probiotic Products. International Journal of Food Microbiology 81 (2003) 1-10.

33. Kirtzalidou E, Pramateftaki P, Kotsou M, Kyriacou A. Screening for lactobacilli with probiotic properties in the infant gut microbiota. Anaerobe 17 (2011) 440-443.

34. Klopper KB, Deane SM, Dicks LMT. Aciduric Strains of Lactobacillus reuteri and Lactobacillus rhamnosus, Isolated from Human Feces, Have Strong Adhesion and Aggregation Properties. Probiotics and Antimicrobial Proteins 10 (2017) 89-97.

35. Olivares M, Diaz-Ropeo MP, Martin R, Rodriguez JM, Xausi J. Antimicrobial potential of four Lactobacillus strains isolated from breast milk. Journal of Applied Microbiology 101 (2006) 72-9. 